\title{
MaxInsTx: A Best-Effort Failure Recovery Approach for Artifact-Centric Business Processes
}

\author{
Haihuan Qin, Guosheng Kang, and Lipeng Guo \\ School of Computer Science, Fudan University, China \\ $\{09110240015,12110240015,10110240002\}$ @fudan. edu.cn
}

\begin{abstract}
Process instances may overlap and interweave with each other. This significantly complicates the failure recovery issue. Most of existing mechanis$\mathrm{ms}$ assume a one-to-one relationship between process instances, which will cause unnecessary recovery in such context. Artifact-centric business process models give equal consideration on both data and control flow of activities, thus facilitate addressing this issue. In this paper, we propose a best-effort failure recovery approach MaxInsTx: a transactional artifact-centric business process model with complex cardinality relationships and correlations considered; a recovery mechanism to resolve the impact of the failed process on concurrent processes meanwhile protect maximal instances involved in failures from failure impact.
\end{abstract}

\section{Introduction}

Business processes (BPs) have become a necessity for modern organizations to stay competitive. In real life, instances of one BP may be split or merged into instances of another BP due to business needs [1-3]. For example, an online shop, acting as a broker, may divide its orders into multiple purchase orders, one pur supplier, and merge items of (different) orders from the same supplier into one purchase order for bulk purchases, as shown in Fig.1. The cardinality between order process and purchase order process is many-to-many and correlations among their instances are complex. We call such scenarios relevant business scenarios. The fabric instances complicates problems and attracts increasing attention [1-3]. No works has been done on the recovery issue yet.

Most of existing workflow transactions simply assume a one-to-one relationship between BP instances (e.g., [4]), and resolve the impact of a failed BP on concurrent BPs through handling dirty reads and dirty writes [5]. Once a failed BP is recovered, all side effects of its committed tasks are semantically undone. However, in the context of relevant business scenario, such recovery is an "over-recovery" and no longer applicable.

Fig. 2 illustrates a relevant business scenario for an online store represented by artifact-centric BP models. This modeling language is used since business data and runtime data gathered in artifacts facilitate modeling instance level correlation [3].

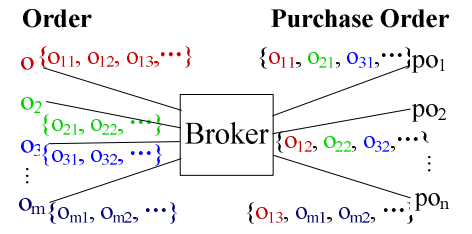

Fig. 1. A relevant business scenario 


\begin{tabular}{|c|c|}
\hline Order & $\mathrm{PO}$ \\
\hline \multirow[t]{3}{*}{\begin{tabular}{l} 
ID \\
state \\
item[] \\
$\quad$ invID \\
name \\
\multicolumn{1}{c}{ quantity } \\
price \\
create \\
customer \\
totalAmt \\
shipment \\
$\ldots$
\end{tabular}} & \begin{tabular}{|l} 
ID \\
state \\
item[] \\
$\quad$ invID \\
$\quad$ name \\
$\quad$ quantity \\
$\quad$ price \\
$\quad$ OrderID \\
vendor \\
$\ldots$ \\
\end{tabular} \\
\hline & SO \\
\hline & $\begin{array}{l}\text { ID } \\
\text { state } \\
\text { item[ } \\
\quad \text { invID } \\
\quad \text { OrderID } \\
\text { address } \\
\ldots\end{array}$ \\
\hline
\end{tabular}

(a) Information models

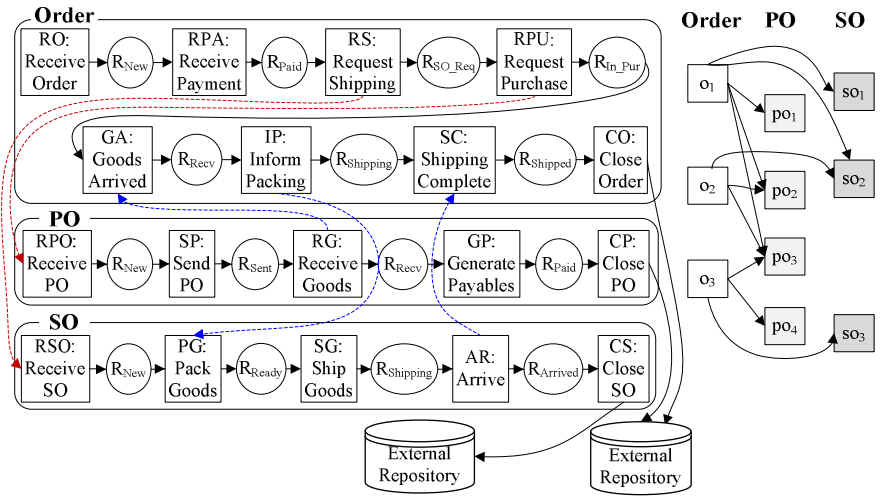

(b) Example workflows of Order, PO and SO

(c) Cardinality

Fig. 2. Workflows involved in the online store example and cardinality between them

Three (key) artifacts are involved: Order, Purchase Order(PO) and Ship Order(SO). Fig. 2(a) shows their information models and Fig. 2(b) their workflows. Attributes of an artifact can be simple or relation-typed (denoted by "[]") with nested attributes. Once receiving a customer order, the store initiates an Order. After receiving payment, the Order create a $P O$ by sending a message to $R P O$, and its task $G A$ cannot be initiated until receives the message from $P O$, i.e., there exist creation and synchronization (sync for short) dependencies between instances of Order and PO. An Order may create multiple $P O s$, one pur supplier, meanwhile multiple Orders may be merged into one $P O$ for bulk purchases. Similar splitting and merge exist among Orders and SOs. The relationships between these artifacts are m-to-m, as shown in Fig. 2(c), and nested attribute $\operatorname{OrderID}$ contained in $P O$ (or $S O$ ) indicates correlations among them.

Several issues are worthy of attention. First, creation dependencies cannot be covered by traditional transactions. For example, an Order $o_{1}$ creates a $P O \mathrm{po}_{1}, \mathrm{po}_{1}$ should be rolled back when $o_{l}$ is canceled, which is similar to dirty reads problem [5]. However, if $p o_{1}$ is rolled back for items are out of stock, $o_{1}$ also needs to be recovered for it cannot be accomplished. Second, it is too costly to recover all dependent artifacts of a failed artifact when dependencies can be m-to-m. For example, if $o_{1}$ is canceled, $p o_{1}, p o_{2}$ and $p o_{3}$ will be affected and recovered, however, the entire recovery of $p o_{2}$ and $p o_{3}$ will further cause other normal cases $o_{2}$ and $o_{3}$ to be affected and recovered. To avoid such over-recovery, the affected parts of $\mathrm{po}_{2}$ and $\mathrm{po}_{3}$ need to be identified, and fixed (i.e., partially rolled back) without affecting $o_{2}$ and $o_{3}$ as well as their purchases.

To solve above questions, we propose a best-effort failure recovery approach MaxInsTx and make two contributions: (1) we develop a transactional artifact-centric BP model with creation dependencies considered and cardinality types of dependencies distinguished; (2) we propose an approach to resolve the impact of the failed BP on concurrent BPs, avoiding unnecessary recoveries through overlap evaluation.

This paper is organized as follows. The transactional model and the failure recovery mechanism are presented in Section 2 and 3 (resp.). Section 4 discusses effectiveness and feasibility. Section 5 discusses related work and Section 6 concludes this paper. 


\section{A Transactional Model for Relevant Artifact-Centric BPs}

\subsection{Preliminaries}

In EZFlow [6], a BP is represented by artifact classes, tasks, repositories and workflow schemas, with a core artifact carrying both data and the enactment. Each artifact class is a tuple $A=$ (name, Atts), where name is its name, Atts is a set of attributes. Each class always contains the attribute $I D$ to hold the unique identifier of its instances.

Definition 2.1: An EZFlow schema is a tuple $(C, \Gamma, R, M, T, F, G)$, where

- $C$ is the core artifact class, $\Gamma$ is a set of auxiliary artifact classes; $R$ is a set of repositories; $M$ is a set of message types; $T$ is a set of tasks;

- $F$ maps each task $t$ in $T$ to a pair $\left(m_{i} ; M_{o}\right)$, where $m_{i}$ is the message type that triggers $t$ and $M_{o}$ is the set of message types produced by $t$. Each message type is produced by at most one task and can be used to trigger at most one task;

- $G$ is a set of triples $(u, v, g)$ where (1) either $u \in R$ and $v \in T$ or $u \in T$ and $v \in R$; and (2) $g$ is a guard on the edge $(u, v)$.

EZFlow uses snapshots to represent the system state of a workflow at time instants, which contains tables for artifact classes, workflow enactments, etc. A workflow moves from one snapshot to another when an external message arrives or a triggered task completes its execution, evolving the artifact along its lifecycle. The enactment of a workflow is an alternating sequence of system states and tasks $s_{0} t_{0} s_{1} t_{1} . . t_{n-1} s_{n}$ such that $s_{0}$ is the initial state and each $s_{i+1}$ is derived from $s_{i}$ through $t_{i}(0 \leq \mathrm{i}<n)$.

\subsection{Correlations and Dependencies of Artifacts}

Correlations combine individual BPs into relevant business scenarios. We use a correlation graph to present correlations between artifacts. Correlation conditions are defined by projection operation $\pi$ and intersection predicate $\Pi$. The projection $\pi_{A_{1}, \ldots, A_{n}}(\alpha)$ restricts artifact $\alpha$ to its attributes set $\left\{A_{1}, \ldots, A_{n}\right\}$. The predicate $\Pi$ checks whether two input sets have overlap. If overlap exists, the expression will be true. An atomic correlation condition is an intersection expression applied to projection expressions.

Definition 2.2: A correlation graph $G$ is a tuple $(\mathcal{A}, E, \Phi, \kappa)$, where

- $\mathcal{A}$ is a nonempty set of artifacts closed under references (through attributes). Artifacts in $\mathcal{A}$ are called nodes of the graph;

- $E \subseteq \mathcal{A} \times \mathcal{A}$ is a set of edges which denotes correlations among artifacts;

- $\Phi: E \rightarrow C o n$ is a mapping that assigns each edge a correlation condition, i.e., a set (conjunction) of atomic conditions; and

- $\kappa: E \rightarrow$ Card is a mapping from $E$ to $\{1: 1,1: \mathrm{m}, \mathrm{m}: 1, \mathrm{~m}: \mathrm{m}\}$ specifying cardinality relationship between artifacts of source node and end node of an edge.

Above definition adapts the correlation graph in [3] to support $m$-to- $m$ cardinality relationship. Instances of two correlated artifacts are correlated if the correlation condition is true on these instances. For example, suppose correlation condition of Order 
and $P O$ is con $=\pi_{I D, \text { item.invID }}($ Order $) \sqcap \pi_{\text {item.orderID, item.invID }}(P O), o_{1}$ and $p o_{1}$ are instances of $\mathrm{Order}$ and $P O$, resp., $o_{l}$ is correlated with $\mathrm{po}_{l}$ if $c o n$ is true on $o_{1}$ and $p o_{l}$.

Only correlated artifacts may exchange messages. Thus, we define messages among artifacts with respect to correlation graph, using "ext" to denote external environment.

Definition 2.3: Given a correlation graph $G=(\mathcal{A}, E, \Phi, \kappa)$, a message type $m s g$ among artifacts wrt $G$ is a tuple $\left(N\right.$, Atts, $\left.\alpha_{s}, \alpha_{r}, \vdash\right)$, where

- $N$ is a distinct message name; Atts is a set of attributes of the message;

- $\alpha_{s}$ and $\alpha_{r}$ denotes the sender and receiver (resp.), satisfying at most one of them can be "ext", and if both are artifacts, they must be correlated;

- $\vdash$ indicates whether the sender creates an instance of the receiving artifact when a message instance arrives, "yes" means creation, "no" means no creation.

Correlated artifacts along with these msgs form a relevant business scenario.

Definition 2.4: A relevant artifact-centric BPs schema $R W$ is a tuple $(G, M s g)$, where $G$ is a correlation graph, and $M s g$ is a set of message types wrt $G$.

Dependencies exist between two artifacts $\alpha_{i}$ and $\alpha_{j}$ if they are correlated. Basicall$\mathrm{y}$, there are two categories of dependencies: creation dependency and sync dependency. $\alpha_{j}$ has creation dependency on $\alpha_{i}$ if $\alpha_{i}$ contributes to the creation of $\alpha_{j}$ (i.e., $\alpha_{i}$ sends a message whose $\vdash$ is "yes" to $\alpha_{j}$ ). $\alpha_{j}$ has sync dependency on $\alpha_{i}$ if $\alpha_{j}$ cannot proceed until the message from $\alpha_{i}$ arrives (i.e., $\alpha_{i}$ sends a message whose $\vdash$ is "no" to $\alpha_{j}$ ).

Instance level dependencies are created at runtime. Given instances $I_{\alpha i}$ of $\alpha_{i}$ and $I_{\alpha j}$ of $\alpha_{j}$, we use notations $\alpha_{j}<I_{\alpha i}>$ and $\alpha_{i} \ll I_{\alpha j} \gg$ to denote the instances of $\alpha_{j}$ which are created by $I_{\alpha i}$ and the instances of $\alpha_{i}$ which contribute to the creation of $I_{\alpha j}$, resp; and $R v\left(I_{\alpha i}, m s g\right)$ and $S d\left(I_{\alpha j}, m s g\right)$ to denote the instances which receive the message $m s g$ sent from $I_{\alpha i}$ and the instances which send the message $m s g$ received by $I_{\alpha j}$, resp. Instance level dependencies are defined as follows.

Definition 2.5: Given artifacts $\alpha_{i}$ and $\alpha_{j}$ and their instances $I_{\alpha i}$ of $\alpha_{i}$ and $I_{\alpha j}$ of $\alpha_{j}$, $I_{\alpha j}$ is creation dependent on $I_{\alpha i}$ if $\alpha_{j}$ is creation dependent on $\alpha_{i}$ and $I_{\alpha j} \in \alpha_{j}<$ $I_{\alpha i}>$. Cardinality type of this dependency is: 1-to-1 if $\left|\alpha_{j}<I_{\alpha i}>\right|=\left|\alpha_{i} \ll I_{\alpha j} \gg\right|=1$; 1-to-m if $\left|\alpha_{j}<I_{\alpha i}>\right|>1$ and $\left|\alpha_{i} \ll I_{\alpha j}\right\rangle \mid=1$; m-to-1 if $\left|\alpha_{j}<I_{\alpha i}>\right|=1$ and $\left|\alpha_{i} \ll I_{\alpha j} \gg\right|>1$; or m-to-m if $\left|\alpha_{j}<I_{\alpha i}>\right|>1$ and $\left|\alpha_{i} \ll I_{\alpha j} \gg\right|>1$.

Definition 2.6: Given artifacts $\alpha_{i}$ and $\alpha_{j}$ and their instances $I_{\alpha i}$ of $\alpha_{i}$ and $I_{\alpha j}$ of $\alpha_{j}$, $I_{\alpha j}$ is sync dependent on $I_{\alpha i}$ if $\alpha_{j}$ is sync dependent on $\alpha_{i}$ and $I_{\alpha j} \in R v\left(I_{\alpha i}, m s g\right)$. Cardinality type of this dependency is: 1-to-1 if $\left|R v\left(I_{\alpha i}, m s g\right)\right|=\left|S d\left(I_{\alpha j}, m s g\right)\right|=1$; 1-to-m if $\left|R v\left(I_{\alpha i}, m s g\right)\right|>1$ and $\left|S d\left(I_{\alpha j}, m s g\right)\right|=1$; m-to-1 if $\left|R v\left(I_{\alpha i}, m s g\right)\right|=1$ and $\left|S d\left(I_{\alpha j}, m s g\right)\right|>1$; or m-to-m if $\left|R v\left(I_{\alpha i}, m s g\right)\right|>1$ and $\left|S d\left(I_{\alpha j}, m s g\right)\right|>1$.

\subsection{EZFlow-Tx: A Transactional Artifact-Centric BP Model}

To avoid unnecessary recovery, we relax the atomicity property: besides "all" or "nothing", we allow an artifact be in a new state "fixed committed", i.e., the artifact is fixed by eliminating its error parts and preserving other parts. 
In detail, we define the following transactional states of an artifact: initial, active, committed, compensated, fixed-running, and fixed committed. An artifact can correctly evolve to state committed, or to state compensated via rolling back operation. Especially, an artifact reaches state fixed-running if some parts of it fail and are fixed. After its execution resumes, it will finally reach state fixed committed. At the same time, we define two transactional states for the relation-typed attributes of an artifact: normal and compensated, representing whether sub-tuples of the attribute is affected by certain errors. Consequently, we add attribute txState to an artifact, and rstate to each relation-type attribute to record their transactional state resp.

Definition 3.7: A transactional artifact-centric BP schema $W$ is a tuple $(E Z, T x)$, where EZ is an EZFlow, and

- $T x$ defines transactional attributes, it maps each task $t$ in $T$ to a tuple (rec, comp, fix) where rec=trivial $\mid$ compensatable, trivial means $t$ has no need to be recovered; and compensatable means the side effects of $t$ can be semantically undone by its compensating task comp or its partial side effects corresponded to the failed parts can be semantically undone through its error fixing task fix after its completion.

In normal execution, the enactment of the transactional workflow is similar to that of EZFLow. The sequence of tasks in the enactment $t_{0} t_{1} . . t_{n-1}$ forms a workflow transaction, wherein each task is a sub-transaction that keeps CID properties. When a task $t$ fails, backward recovery will be initiated unless task $t$ is trivial.

Now we describe the recovery procedure. When an artifact $I_{f}$ fails and is recovered, its dependent artifacts will be identified. Suppose artifact $I_{r}$ depends on $I_{f}$ with the cardinality type of m-to- 1 or m-to-m, i.e., $I_{f}$ only contributes to parts of $I_{r}$, we will (1) invoke the fixing task fix of $I_{r}$ to fix the parts affected by $I_{f}$ and preserve unaffected parts. After fixing, sub-transaction of fixed tasks are set to fixed committed, rstate of the fixed sub-tuples are set to compensated, and txState of $I_{r}$ is set tofixed-running; then (2) resume the execution of $I_{r}$ to handle its normal parts upon recovery completion. If the executions of rest tasks of $I_{r}$ are successfully committed, txState of $I_{r}$ will be set to fixed committed. Note that cardinality and correlations are used to determine the failure parts of $I_{r}$. If an artifact is entirely affected, it will be wholly compensated, the txState of the artifact together with the rstate of all its sub-tuples will be set to compensated.

The transaction of a relevant scenario is correct if every failed transaction has been semantically undone or fixed, and artifacts, which have creation/created or sync dependency on it, have also been semantically undone or fixed.

\section{A Recovery Mechanism for Relevant BPs}

Here, we outline a creation/sync dependency discovery method and a recovery mechnism for keeping data consistency of relevant business scenario when failure occurs.

\subsection{Creation/Sync Dependency Discovery and Overlaps}

To discover dependencies among artifacts, we design two relations: (1) instances creation record InstCre(cre, cred, $m s g$, $p d T, r v T, t s$ ) where cre is an artifact which sends the 
message msg through task $p d T$, cred is an artifact created by task $r v T$ at the time the $m s g$ arrives, $t s$ represent the execution time of $r v T$; (2) instances sync record SynDepd $(s d, s y n, m s g, p d T, r v T, t s)$ where $s d$ and $s y n$ are artifacts that sends and receives the message $m s g$ resp. When a task is triggered by a message $m s g$, a tuple will be added into InstCre if $m s g$ has "yes" as value of $\vdash$, otherwise, a tuple will be added into SynDepd.

When an instance $I_{i}$ of artifact $\alpha_{i}$ fails and is recovered, its dependent artifacts can be derived as follows: (1) artifacts with creation dependency: $\pi_{\text {cred }}\left(\sigma_{\text {cre }=I_{i}}(\right.$ InstCre $\left.)\right)$; (2) artifacts with created dependency: $\pi_{c r e}\left(\sigma_{\text {cred }=I_{i}}(\right.$ InstCre $\left.)\right)$; and (3) artifacts with sync dependency: $\pi_{s y n}\left(\sigma_{s d=I_{i}}(\right.$ SynDepd $\left.)\right)$. For every dependent instance $I_{x}$, we can similarly compute the number of artifacts on which it is creation/created or sync dependent, thus, the cardinality type of dependency between $I_{i}$ and $I_{x}$ can be determined.

When cardinality type of dependency between $I_{x}$ and $I_{i}$ is not 1-to-1, we need to fix the error parts of $I_{x}$ which overlap $I_{i}$ rather than wholly roll back. The error parts can be computed by $\sigma_{\varphi}\left(\alpha_{x}\right)$, where $\alpha_{x}$ is the artifact class of $I_{x}$, and $\varphi$ is constructed as follows. First, get correlation condition between $\alpha_{x}$ and $\alpha_{i}$ from correlation graph $G$, construct an equation expressions for each pair of projection attributes of each intersection expression in turn. Then construct two equation expressions $\alpha_{x} . I D=I_{x}$ and $\alpha_{i} . I D=I_{i}$ to filter the result. $\varphi$ is a conjunction of all these equation expressions.

For example, when the Order instance $o_{3}$ in Fig. 2(c) fails, $p o_{3}$ will need to be fixed for $p o_{3} \in P O<o_{3}>$ and cardinality type of the dependency is m-to-1. Suppose Order and $P O$ are correlated and the correlation condition is $\pi_{I D \text {, item.invID }}($ Order $) \Pi$ $\pi_{\text {item.orderID, item.invID }}(P O)$. The failed parts of $p o_{3}$ can be computed by $\sigma_{P O . I D}=p o_{3} \wedge P O$. item.OrderID=Order.ID $\wedge P$ O.item.invID=Order.item.invID $\wedge$ Order.ID $=o_{3}(P O)$.

\subsection{A Mechanism for Handling Cascaded Recovery}

Cascaded recovery needs to be considered because isolation is relaxed. When an instance $I_{i}$ fails, its dependent instances $I_{D}$ will be identified. However, dependencies do not necessarily indicate being affected. To avoid over-recovery, we use overlaps to determine if an instance $I_{d} \in I_{D}$ is really affected. Different overlap degrees have different recovery strategies: (1) no overlap: resume its execution; (2) complete overlap: entirely recover it; (3) partial overlap: fix the parts that overlap the failed instance, then resu- me its execution to handle the rest normal parts upon recovery completion. When $I_{d}$ needs to be recovered, it may cause its dependent instances to be cascaded recovered.

We construct a directed acyclic graph Artifact Dependency Graph (ADG) for $I_{i}$, containing all its dependent instances which overlap its failed parts. Each node in an ADG represents an artifact. Its structure contains: (1) cID: artifact identifier, denoted by $I_{i}$; (2) deFrom $\left\langle c I D\right.$, type $>$ : a list records artifacts that directly cause $I_{i}$ to be recovered and corresponding affecting type; (3) failedPart: the failed parts of $I_{i}$; and (4) bkRecCmd: recovery command for $I_{i}$, which can be "compensate" or "fix". Each edge represents dependency between nodes with solid line for creation dependency and dash line for sync dependency. If a node $I_{x}$ depends on node $I_{y}$, there will be an edge from $I_{x}$ to $I_{y}$. The structure of an edge is a tuple $e=$ (source, target, type) where type describes dependency type between source and target nodes, i.e., "create", "created" or "sync". 
When building an ADG for the failed instance $I_{f}$, instances with creation/created or sync dependency on $I_{f}$ are identified, and those ones overlap its failed parts are added into the graph. For a creation dependent instance $I_{c d}$, only instances that have sync or creation dependencies on it meanwhile overlap its failed parts are affected and need to be added. For a created dependent instance $I_{c}$, only instances which have sync or created dependencies on it meanwhile overlap its failed part need to be added. The recovery of a sync dependent instance $I_{s d}$ is similar to that of the failed instance.

If $I_{i}$ is affected by one instance, its failedPart is the intersection between $I_{i}$ and the failedPart of that instance. If $I_{i}$ is affected by multiple instances, overlaps between $I_{i}$ and these instances will be computed resp., and the failedPart is the union of these overlaps. With regard to instances that may depend on each other, we use the following rules to avoid creating a cycle. Given two nodes $I_{x}$ and $I_{y}, I_{y}$ is dependent on $I_{x}$ and overlaps its failedPart. $I_{y}$ will be added as a dependent instance of $I_{x}$ if $I_{y}$ has not existed in the ADG. Otherwise, missing nodes affected by $I_{y}$ should be added, and failedPart should be adjusted for $I_{y}$ and all instances derived from it. For each derived instance $I_{z}$ with creation type, if overlap between failedPart of $I_{y}$ and $I_{z}$ contains $I_{z}$ 's failedPart, similar failedpart adjustment should be done for $I_{z}$. The overlap adjustment is iteratively handled until there is no change to the intersection or there is no overlap with other instances. In this way, cycling dependency is avoided, and the recovery of $I_{y}$ does not miss any affected parts since the impact of $I_{x}$ 's failure on $I_{y}$ has been handled.

The ADG construction algorithm builds an ADG for the failed instance $I_{f}$. The algorithm has two inputs: $I_{f}$ and its fail parts failedPart. An ADG is represented by a set of nodes $N$ and a set of edges $E$. The algorithm is outlined as follows.

1. Create two assisting lists: (1) affLi<aff, deFrom, $d p>$ to store affected instances and iterate through different level of a ADG, where aff is derived from deFrom with dependency type $d p$; (2) tempLi to temporarily store the affected instances of instances in affLi.

2. Construct a node for $I_{f}$ and add it into affLi to start ADG construction.

3. For each element $e l$ in affLi,

3.1. If el.aff does not exist in $N$, add an edge from el.deFrom to el.aff into $E$, add every affected instances of el.aff into tempLi.

3.2. Else retrieve el.aff into $I_{m}$ (affected by multiple instances. No cycle). If NotContain ( $I_{m}$.deFrom.type, el.dp) or NotEmptyDifferenceSet(el.aff.failedPart, $I_{m}$.failedPart), add missing instances affected by el.aff into tempLi, adjust failedpart of all affected instances.

4. After processing all elements of affLi, empty aff $L i$ and move instances from tempLi to affLi to start next level construction. If $a f f L i$ is not empty, repeat step 3.

\section{Fig. 3 shows a sample $\mathrm{ADG}_{1}$ for $I_{f}$ containing ten concurrent BPs $I_{1}-I_{10}$.}

The ADG is used to conduct cascaded recovery. When a BP $I_{f}$ fails, its dependent BPs will be identified and suspended for overlap evaluation, while independent BPs keep running. Then the recovery procedure invokes the ADG construction algorithm to construct an ADG for $I_{f}$, with failed parts of each affected instance computed, and finally traverse the ADG to recover each affected instance $I_{a}$ according to its recovery command bkRecCmd, which is set to "compensate" when its failed parts equal to its contents, or set to "fix" when its failed parts are a proper subset of its contents. Due to space limit, algorithm details are ignored here. 


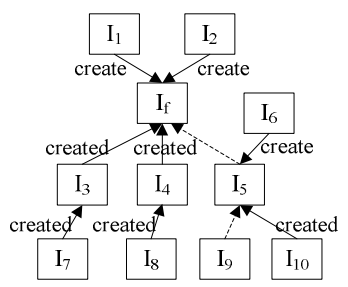

Fig. 3. Sample $\mathrm{ADG}_{1}$ for $I_{f}$
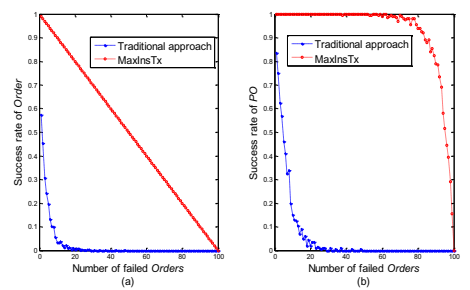

Fig. 4. Success rate comparison

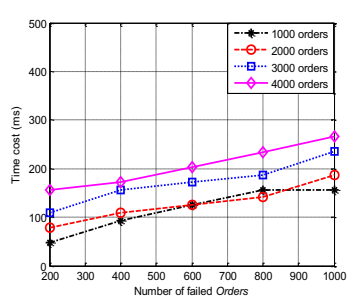

Fig. 5. Time cost

\section{$4 \quad$ Evaluation of Effectiveness and Feasibility}

To evaluate the effectiveness, we compare the BP success rate of MaxInsTx with that of traditional approach (all or nothing). Experiment is conducted on $m$ Orders and $n$ $P O s$, where $m=100, n=20$. We assign [1,5] items to each Order with uniform distribution. The probability that an item in an Order belongs to a certain $P O$ is $1 / \mathrm{n}$.

Fig. 4 shows the success rate of Orders and POs. In Fig. 4(a), the success rate of Orders of MaxInsTx decrease linearly because only the failed Orders will fail. In Fig. 4(b), the success rate of POs of MaxInsTx shows no impact when the number of failed Orders varies from 1 to 54, because POs will fail only when they become empty due to the failed Orders. After that, the SRPO decreases gradually for a while and then decreases dramatically in the last period. However, both the success rate of Orders and $P O s$ of traditional approach decrease dramatically, since other Orders and all the POs correlated with the failed Orders will fail. Obviously, MaxInsTx has much better performance, which verifies its effectiveness.

Next, we analyze the time cost of MaxInsTx. In this experiment, the number of failed Orders varies from 200 to 1000, the number of total Orders varies from 1000 to 4000 , and the number of $P O s$ is fixed to 20 . The configuration of our microcomputer is as follows: 3.2 GHz Dual Core processor, 2 GB memory, Windows XP OS.

Fig. 5 shows that the time cost is affected by both the number of failed Orders and total Orders. The time cost curve increases slowly with the increasing of the number of failed Orders. The overall time cost is very small. Hence MaxInsTx is feasible.

\section{$5 \quad$ Related Work}

Many works have addressed the issue of long-live transaction coordination through ACID relaxation. One of the representative approaches is proposed in [4] which allows relaxing any attributes of ACID through introducing a "pre-commit" phase and a "negotiation" phase. Xiao et al [5] further address the issue of concurrency control. They propose a rule-based approach to resolve the concurrency issue through analyzing write dependency and potential read dependency among BPs.

None of the above works take the impact of complex cardinality relationships into consideration. In practice, the existing of m-to-m relationships among BPs complicates many problems and attracts increasing attention. Fahland et al [1] address the behavioral conformance checking problem complicated by the fabric of BP instances. Zhao et al [2] focus on managing instance correspondence through correlations attached to each 
instance. Sun et al [3] develop a choreography language which supports instance level correlations and cardinality constraints. Different from these works, our research focuses on solving the recovery issue for the fabric BPs. A recovery approach is proposed to resolve the impact of a failed BP on concurrent BPs meanwhile avoid over-recovery.

\section{Conclusion}

This paper proposed a best-effort failure recovery approach MaxInsTx for relevant business scenarios, supporting complex dependencies between artifacts. We relax the atomicity property of transactions, allowing an artifact be partially fixed such that its unaffected parts are preserved as much as possible. A failure recovery mechanism is proposed with the advantage of avoiding unnecessary recoveries.

Acknowledgment. This work is partially supported by NSFC grant 60873115 .

\section{References}

1. Fahland, D., de Leoni, M., van Dongen, B.F., van der Aalst, W.M.P.: Conformance Checking of Interacting Processes with Overlapping Instances. In: Rinderle-Ma, S., Toumani, F., Wolf, K. (eds.) BPM 2011. LNCS, vol. 6896, pp. 345-361. Springer, Heidelberg (2011)

2. Zhao, X., Liu, C., Yang, Y., Sadiq, W.: Handling instance correspondence in inter-organisational workflows. In: Krogstie, J., Opdahl, A.L., Sindre, G. (eds.) CAiSE 2007. LNCS, vol. 4495, pp. 51-65. Springer, Heidelberg (2007)

3. Sun, Y., Xu, W., Su, J.: Declarative Choreographies for Artifacts. In: Liu, C., Ludwig, H., Toumani, F., Yu, Q. (eds.) ICSOC 2012. LNCS, vol. 7636, pp. 420-434. Springer, Heidelberg (2012)

4. Khachana, R.T., James, A., Iqbal, R.: Relaxation of acid properties in AuTra, the adaptive user-defined transaction relaxing approach. Future Gener. Comput. Syst. 27(1), 58-66 (2011)

5. Xiao, Y., Urban, S.D.: Using rules and data dependencies for the recovery of concurrent processes in a service-oriented environment. IEEE Transactions on Services Computing 5(1), 59-71 (2012)

6. Xu, W., Su, J., Yan, Z., Yang, J., Zhang, L.: An Artifact-Centric Approach to Dynamic Modification of Workflow Execution. In: Meersman, R., et al. (eds.) OTM 2011, Part I. LNCS, vol. 7044, pp. 256-273. Springer, Heidelberg (2011) 